\title{
Computing three-dimensional two-phase flows with a mass-conserving level set method
}

\author{
S. P. van der Pijl · A. Segal · C. Vuik · P. Wesseling
}

Received: 9 October 2007 / Accepted: 9 January 2008 / Published online: 28 March 2008

(C) The Author(s) 2008

\begin{abstract}
A method is described to compute threedimensional two-phase flow, allowing large density ratios and coalescence and break-up of bubbles. The level set method is used to describe interfaces, and the volume-offluid method is used to ensure mass conservation. Efficiency in computing the interface dynamics is achieved by using a functional relation between the level set and volume-of-fluid functions. Difficulties and remedies in re-initialization of the level set function and inaccurate compution of surface tension are discussed. Test cases for validation are described, and demanding two-bubble computations to show the generality and the versatility of the method are presented.
\end{abstract}

Dedicated to Wolfgang Hackbusch on the occasion of his 60th birthday.

Communicated by G. Wittum.

Supported by the Netherlands Organization for Scientific Research (NWO).

\section{S. P. van der Pijl}

Centrum voor Wiskunde en Informatica (CWI),

The Dutch National Research Centre

for Mathematics and Computer Science,

P.O. Box 94079, 1090 GB

Amsterdam, The Netherlands

\author{
A. Segal · C. Vuik $(\varangle) \cdot$ P. Wesseling \\ Faculty of Technical Mathematics and Informatics, \\ Delft University of Technology, \\ Mekelweg 4, 2628 CD Delft, The Netherlands \\ e-mail: c.vuik@tudelft.nl \\ P. Wesseling \\ e-mail: p.wesseling@tudelft.nl
}

\section{Introduction}

Computation of multiphase flows from first principles is intractable, because of the occurrence of very many interfaces; think for example of a mixture of air bubbles and water (or better still beer). Hence, for industrial applications heuristic models are used. To validate and refine these models, information from physical experiment and computation from basic principles is required. However, even for flows with a small or moderate number of bubbles some heuristic modelling is required to make computation feasible, even with efficient parallel solvers. The challenge is to keep the model as accurate as possible without sacrificing too much computing time. Here we present a contribution to computation of two-phase flows.

Capturing of interfaces between phases is easily and efficiently done with the level set (LS) method $[14,16,22]$. It does not conserve mass, however, which is the strong point of the volume-of-fluid (VOF) method [29,9,18,19], which is another prominent method to track fluid interfaces. Mass errors of the level set method can be reduced by increasing the order of discretization and/or utilizing adaptive mesh refinement near the interface, see e.g. [6,7,13]. Olsson et al. [15] choose a VOF-like level set function and base their method directly on conservation laws. To make the LS method massconserving, some methods couple LS and VOF, such as the CLSVOF method [21,24], its adaptive variant (ACLSVOF) [32] and our MCLS method [27,28]. The special feature of MCLS is, that an explicit relation between the LS and VOF functions is exploited to find small mass-conserving corrections to the LS function with small computing cost. Here we present the extension of MCLS to three dimensions and surface tension.

Surface tension depends on the curvature of the interface. The small corrections to the LS function that were just 
mentioned make the interface wrinkly and inhibit accurate determination of interface curvature. Parasitic currents result; these are also generated by the re-initializations required to let the LS function remain a distance function. A curvature smoothing and a re-initialization method are devised that make parasitic currents convergence to zero with the mesh size. Another issue is occurrence of under- and overshoots in the VOF function. We counter this with a mass-redistribution approach. These measures and the extension to 3D flows with surface tension constitute the novelty of this paper compared to $[27,28]$. Applications to rising bubbles and falling droplets will be presented.

\section{Time-stepping with the pressure-correction method}

Consider flow of two phases with constant density and viscosity, separated by an interface $S$, possibly consisting of disjoint parts. It is described by a level set function $\Phi$, as follows: $S(t)=\left\{\mathbf{x} \in \mathbb{R}^{3} \mid \Phi(\mathbf{x}, t)=0\right\}$. The interface boundary conditions express continuity of mass and momentum at the interface (which can be found in any relevant textbook, but see e.g. Tryggvason et al. [26] for a recent reference):

$$
\begin{aligned}
& {[\mathbf{u}]=0} \\
& {\left[p \mathbf{n}+\mu\left(\nabla \mathbf{u}+\nabla \mathbf{u}^{t}\right) \cdot \mathbf{n}\right]=\sigma \kappa \mathbf{n}}
\end{aligned}
$$

where the brackets denote jumps across the interface, $\mathbf{u}$ is the velocity vector, $p$ the pressure, $\mathbf{n}$ is a normal vector at the interface, $\sigma$ is the surface tension coefficient and $\kappa$ is the curvature of the interface. The interface conditions (2) show that the jump conditions for pressure and velocity are coupled. Furthermore, Li and Lai [12] show that although the velocity is continuous at the interface, the velocity gradients are not. If $\mathbf{s}$ is a vector parallel to the interface, $u_{n}=\mathbf{n} \cdot \mathbf{u}$ is the interface normal velocity component and $u_{s}=\mathbf{s} \cdot \mathbf{u}$ a velocity component parallel to the interface, then

$$
\begin{aligned}
& {\left[\frac{\partial u_{n}}{\partial n}\right]=0, \quad\left[\frac{\partial u_{n}}{\partial s}\right]=0,} \\
& {\left[\frac{\partial u_{s}}{\partial n}\right]=-[\mu] \frac{\partial u_{n}}{\partial s},\left[\frac{\partial u_{s}}{\partial s}\right]=0 .}
\end{aligned}
$$

But note that if the viscosity $\mu$ is continuous at the interface, Eq. (3) shows that the derivatives of the velocity components are continuous too. In that case Eq. (2) reduces to

$$
\begin{aligned}
& {[\nabla \mathbf{u}]=\mathbf{0},} \\
& {[p]=\sigma \kappa .}
\end{aligned}
$$

This means that, besides that the velocity gradients are continuous at the interface, the jump conditions for pressure and velocity are decoupled, which are two major advantages for the numerical treatment of the moving boundary problem.
The jump in viscosity $\mu$ over $S$ is smeared out over three mesh widths, as in $[10,12]$, to make $\mu$ continuous, thereby simplifying the jump conditions across $S$ resulting from conservation of mass and momentum:

$[\mathbf{u}]=\mathbf{0}$ and $[p]=\sigma \kappa$.

The jump condition for $p$ is implemented by means of the continuous surface force method [2]. This means that the jump condition for the normal stresses are replaced by a body force given by

$\mathbf{f}=-\frac{1}{\bar{\rho}} \sigma \kappa \delta_{\alpha}(\Phi) \nabla \Phi$

where $\bar{\rho}$ is the average density and $\delta_{\alpha}$ is a smeared-out delta function; for more details, see [28].

With these approximations, each phase is governed by the incompressible Navier-Stokes equations with the above body force:

$$
\begin{aligned}
\frac{\partial \mathbf{u}}{\partial t}+\mathbf{u} \cdot \nabla \mathbf{u} & =-\frac{1}{\rho} \nabla p+\frac{1}{\rho} \nabla \cdot \mu\left(\nabla \mathbf{u}+\nabla \mathbf{u}^{t}\right)+\mathbf{f}+\mathbf{g}, \\
\nabla \cdot \mathbf{u} & =0,
\end{aligned}
$$

where $\mathbf{g}$ is the acceleration of gravity.

Discretization takes place on a three-dimensional Cartesian staggered grid in the usual way, as described in $[28,31]$. For brevity we use the same symbols for spatial difference and differential operators. Time stepping takes place with the pressure-correction method [30]. First, a tentative velocity vector $\mathbf{u}^{*}$ is computed by:

$\frac{\mathbf{u}^{*}-\mathbf{u}^{n}}{\Delta t}=-\nabla \cdot \mathbf{u}^{n} \mathbf{u}^{n}+\frac{1}{\rho} \nabla \cdot \mu\left(\nabla \mathbf{u}^{*}+\left(\nabla \mathbf{u}^{n}\right)^{t}\right)$,

where superscript $n$ denotes time-level $n$. The stress tensor is split in an implicit part (superscript $*$ ) and an explicit part (superscript $n$ ) to uncouple the velocity components and to obtain symmetric systems that are conveniently solved with the ICCG method. The pressure correction is given by

$\mathbf{u}^{n+1}-\mathbf{u}^{*}=\Delta t\left(-\frac{1}{\rho} G p+\mathbf{g}+\mathbf{f}\right)$,

where $G$ is the discretization of the gradient operator. Discretization of the divergence constraint gives $D \mathbf{u}^{n+1}=f_{b}$ where $f_{b}$ arises from the boundary conditions. Application to Eq. (10) gives the following equation for the pressure:

$D \frac{1}{\rho} G p=D\left(\frac{1}{\Delta t} \mathbf{u}^{*}+\mathbf{g}+\mathbf{f}\right)-f_{b}$

Discretization of $\frac{1}{\rho} \nabla p$ at the interface $S$ requires special care, because $\rho$ is discontinuous at $S$. Use is made of $\left[\frac{1}{\rho} \nabla p\right]=\mathbf{0}$, 
which results from the use of the CSF approximation for the surface tension. A typical example is:

$$
\begin{aligned}
& \left(\beta \frac{\partial p}{\partial x}\right)_{i+1, j+\frac{1}{2}, k+\frac{1}{2}} \approx \\
& \quad \hat{\beta}_{i+1, j+\frac{1}{2}, k+\frac{1}{2} \frac{p_{i+\frac{3}{2}, j+\frac{1}{2}, k+\frac{1}{2}}-p_{i+\frac{1}{2}, j+\frac{1}{2}, k+\frac{1}{2}}}{\Delta x},}
\end{aligned}
$$

where $\hat{\beta}$ is the harmonic average of $\beta \equiv 1 / \rho$. Equation 11 is solved by the ICCG method.

Generalization of the stability restrictions on the time step given in [28] from two to three dimensions is straightforward. Following [10,23], time step bounds related to convection $\Delta t_{c}$ and to surface tension $\Delta t_{s}$ separately are derived, and the following heuristic stability condition is applied:

$\Delta t \leq \operatorname{CFL} \min \left(\Delta t_{c}, \Delta t_{s}\right)$,

where we use $\mathrm{CFL}=1 / 2$, following $[10,23]$. We have

$\Delta t_{c}=\frac{1}{\frac{|u|_{\max }}{\Delta x}+\frac{|v|_{\max }}{\Delta y}+\frac{|w|_{\max }}{\Delta z}}$.

For $\Delta t_{s},[10]$ gives, in two dimensions,

$\Delta t_{s}=\left(\frac{\min \left(\rho_{0}, \rho_{1}\right) \min (\Delta x, \Delta y)^{2}}{\sigma|\kappa|_{\max }}\right)^{1 / 2}$,

where subscripts 0 and 1 refer to the two phases. Because we regularize the surface tension, we replace $\sigma \kappa / \rho$ by $2 \sigma \kappa \delta$ $(\Phi) h /\left(\rho_{0}+\rho_{1}\right)$ and replace Eq. (15) by, in three dimensions:

$\Delta t_{s}=\left(\frac{\left(\rho_{0}+\rho_{1}\right) \min (\Delta x, \Delta y, \Delta z)}{2|\sigma \kappa \delta(\Phi)|_{\max }}\right)^{1 / 2}$.

\section{Interface dynamics}

The dynamics of the interface $S(t)$ are primarily handled with the level set method, details of which may be found in $[3,14,16,28,22,25]$. We have

$S(t)=\left\{\mathbf{x} \in \mathbb{R}^{3} \mid \Phi(\mathbf{x}, t)=0\right\}$,

where the level set function $\Phi$ satisfies

$\frac{\partial \Phi}{\partial t}+\mathbf{u} \cdot \nabla \Phi=0$

While stepping forward in time, $\mathbf{u}$ and $\Phi$ are updated in alternating order. To conserve mass, corrections are applied to $\Phi$ using the volume-of-fluid method. The VOF function $\Psi$ measures the volume fraction of one of the phases in each cell. Call the result of a time step with Eq. (18) $\Phi^{*}$, starting from time level $n+1 / 2$. A correction to $\Phi^{*}$ is determined in three steps:

1. Determine $\Psi^{n+1 / 2}=f\left(\Phi^{n+1 / 2}, \nabla \Phi^{n+1 / 2}\right)$;

2. Use the VOF method to find $\Psi^{n+3 / 2}$;

3. Find a correction $\delta \Phi$ such that $\Phi^{n+3 / 2}=\Phi^{*}+\delta \Phi$ satifies $f\left(\Phi^{n+3 / 2}, \nabla \Phi^{n+3 / 2}\right)=\Psi^{n+3 / 2}$,

where $f$ is explained hereafter and computes the volume of fluid function $\Psi$ based on a linearization of $\Phi$. The steps are now described in more detail.

\subsection{Relation between level set and volume-of-fluid functions}

The VOF function is the volume fraction of one the phases in each cell, and hence is a number assigned to each cell. Given $\Phi$, it can be defined as

$\Psi_{k}=\frac{1}{\operatorname{vol}\left(\Omega_{k}\right)} \int_{\Omega_{k}} H(\Phi) \mathrm{d} \Omega$,

where $\Omega_{k}$ is a cell in the grid and $H$ is the Heaviside step function. We wish to determine a function $f$ such that $\Psi_{k} \approx$ $f\left(\Phi_{k}, \nabla \Phi_{k}\right)$. Let $\varphi$ be the linearization of $\Phi$ at the cell center $\mathbf{x}_{k}$ :

$\varphi\left(\mathbf{x} ; \Phi, \nabla \Phi, \mathbf{x}_{k}\right)=\Phi_{k}+\nabla \Phi \cdot\left(\mathbf{x}-\mathbf{x}_{k}\right)$,

where we approximate $\nabla \Phi$ by central differences, using neighboring cells. The volume fraction $\Psi_{k}$ cut from cell $\Omega_{k}$ by the plane defined by $\varphi=0$ is computed as follows. Map $\Omega_{k}$ onto the unit cube with coordinates $(\xi, \eta, \zeta) \in(-1 / 2,1 / 2)^{3}$, and rewrite Eq. (20) as

$\varphi=\Phi_{k}+D_{\xi} \xi+D_{\eta} \eta+D_{\zeta} \zeta$

and choose the axes such that

$D_{\xi} \geq D_{\eta} \geq D_{\zeta} \geq 0$

Hence,

$$
\begin{aligned}
D_{\xi}= & \max \left(\left|\Delta x\left(\frac{\partial \Phi}{\partial x}\right)_{k}\right|,\left|\Delta y\left(\frac{\partial \Phi}{\partial y}\right)_{k}\right|,\left|\Delta z\left(\frac{\partial \Phi}{\partial z}\right)_{k}\right|\right), \\
D_{\zeta}= & \min \left(\left|\Delta x\left(\frac{\partial \Phi}{\partial x}\right)_{k}\right|,\left|\Delta y\left(\frac{\partial \Phi}{\partial y}\right)_{k}\right|,\left|\Delta z\left(\frac{\partial \Phi}{\partial z}\right)_{k}\right|\right), \\
D_{\eta}=\left|\Delta x\left(\frac{\partial \Phi}{\partial x}\right)_{k}\right|+\left|\Delta y\left(\frac{\partial \Phi}{\partial y}\right)_{k}\right| & +\left|\Delta z\left(\frac{\partial \Phi}{\partial z}\right)_{k}\right|-D_{\xi}-D_{\zeta} .
\end{aligned}
$$


Because of symmetry, it suffices to consider the case $\Phi \leq 0$. Distinguish the following two cases:

case I : $D_{\xi} \leq D_{\eta}+D_{\zeta}$,

case II : $D_{\xi}>D_{\eta}+D_{\zeta}$.

These two cases are illustrated in Fig. 1. The volume cut out from the cube can be found by subtracting the volumes of the shaded tetraeders from the large tetraeder. Length, width and height of these tetraeders are $\Phi_{A} / D_{\xi}, \Phi_{A} / D_{\eta}, \Phi_{A} / D_{\zeta}$, and similarly for the other vertices. We have

$$
\begin{aligned}
& \Phi_{A}=\Phi_{k}+\left(D_{\xi}+D_{\eta}+D_{\zeta}\right) / 2, \\
& \Phi_{B}=\Phi_{k}+\left(D_{\xi}+D_{\eta}-D_{\zeta}\right) / 2, \\
& \Phi_{C}=\Phi_{k}+\left(D_{\xi}-D_{\eta}+D_{\zeta}\right) / 2, \\
& \Phi_{D}=\Phi_{k}+\left(-D_{\xi}+D_{\eta}+D_{\zeta}\right) / 2, \\
& \Phi_{E}=\Phi_{k}+\left(D_{\xi}-D_{\eta}-D_{\zeta}\right) / 2 .
\end{aligned}
$$

We find:

$f= \begin{cases}\frac{\mathcal{A}}{6 D_{\xi} D_{\eta} D_{\zeta}}, & \Phi \leq 0, \\ 1-f(-\Phi, \nabla \Phi), & \Phi>0,\end{cases}$

where

$$
\begin{aligned}
\mathcal{A}= & \max \left(\Phi_{A}, 0\right)^{3}-\max \left(\Phi_{B}, 0\right)^{3}-\max \left(\Phi_{C}, 0\right)^{3} \\
& -\max \left(\Phi_{D}, 0\right)^{3}+\max \left(\Phi_{E}, 0\right)^{3} .
\end{aligned}
$$

3.2 Time advancement of volume-of-fluid function

The flux-splitting of [24] is adopted:

$$
\begin{gathered}
\Psi_{i, j, k}^{(x)}=\frac{\Psi_{i, j, k}^{n+1 / 2}-\frac{1}{\Delta x \Delta y \Delta z}\left(F_{x_{i+\frac{1}{2}, j, k}}^{n+1 / 2}-F_{x_{i-\frac{1}{2}, j, k}}^{n+1 / 2}\right)}{1-\frac{\Delta t}{\Delta x}\left(u_{i+\frac{1}{2}, j, k}-u_{i-\frac{1}{2}, j, k}\right)}, \\
\Psi_{i, j, k}^{(y)}=\frac{\Psi_{i, j, k}^{(x)}-\frac{1}{\Delta x \Delta y \Delta z}\left(F_{y_{i, j+\frac{1}{2}, k}}^{(x)}-F_{y_{i, j-\frac{1}{2}, k}}^{(x)}\right)}{1-\frac{\Delta t}{\Delta y}\left(v_{i, j+\frac{1}{2}, k}-v_{i, j-\frac{1}{2}, k}\right)}, \\
\Psi_{i, j, k}^{(z)}=\frac{\Psi_{i, j, k}^{(y)}-\frac{1}{\Delta x \Delta y \Delta z}\left(F_{z_{i, j, k+\frac{1}{2}}}^{(y)}-F_{z_{i, j, k-\frac{1}{2}}}^{(y)}\right)}{1-\frac{\Delta t}{\Delta z}\left(w_{i, j, k+\frac{1}{2}}-w_{i, j, k-\frac{1}{2}}\right)}, \\
\Psi_{i, j, k}^{n+3 / 2}=\Psi_{i, j, k}^{(z)}-\Delta t\left(\Psi_{i, j, k}^{(x)} \frac{u_{i+\frac{1}{2}, j, k}-u_{i-\frac{1}{2}, j, k}}{\Delta x}\right. \\
+\Psi_{i, j, k}^{(y)} \frac{v_{i, j+\frac{1}{2}, k}-v_{i, j-\frac{1}{2}, k}}{\Delta y} \\
\left.+\Psi_{i, j, k}^{(z)} \frac{w_{i, j, k+\frac{1}{2}}-w_{i, j, k-\frac{1}{2}}}{\Delta z}\right),
\end{gathered}
$$

where the fluxes $F$ represent the fractional volumes that flow into or out of a cell through the face under consideration.

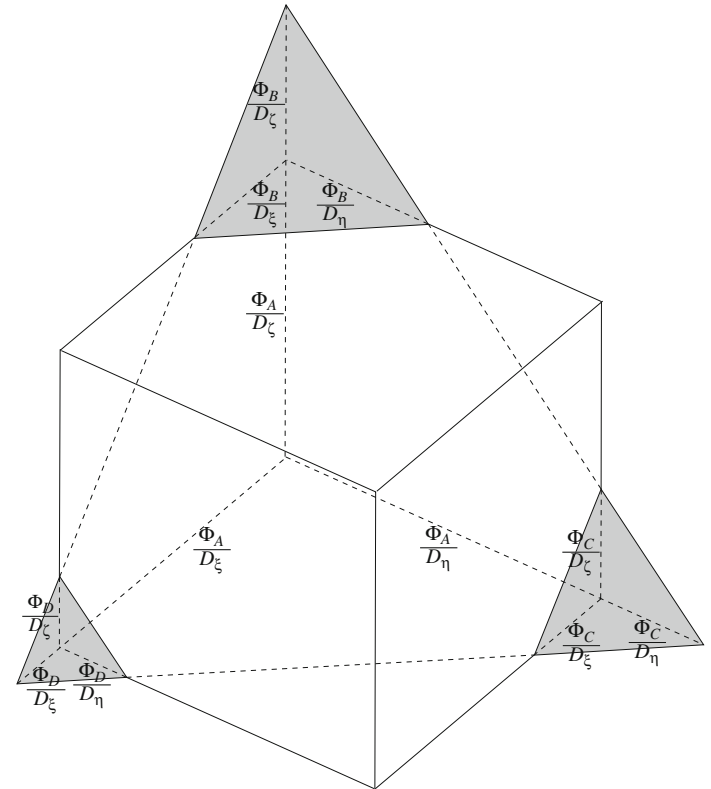

(a) case I

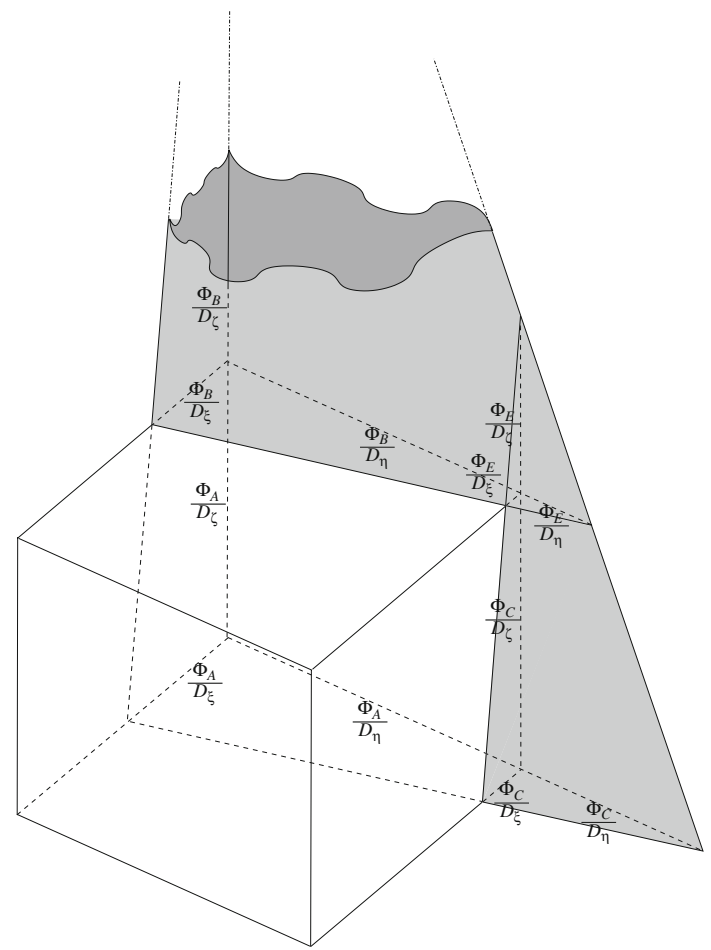

(b) case II

Fig. 1 Volumes for $\Phi \leq 0$

The fluxes are determined from the level set function $\Phi$ by Eq. (26). For example, $F_{z}=F_{z}^{+}+F_{z}^{-}$, with

$$
\begin{aligned}
& F_{z i, j, k+\frac{1}{2}}^{+}=\Delta V v^{+} f\left(\widehat{\Phi}_{L},\left(\partial_{x} \Phi_{L}, \partial_{y} \Phi_{L}, v^{+} \partial_{z} \Phi_{L}\right)^{t}\right), \\
& F_{z i, j, k+\frac{1}{2}}^{-}=\Delta V v^{-} f\left(\widehat{\Phi}_{R},\left(\partial_{x} \Phi_{R}, \partial_{y} \Phi_{R},-v^{-} \partial_{z} \Phi_{R}\right)^{t}\right),
\end{aligned}
$$


where $\Delta V=\Delta x \Delta y \Delta z, \Phi_{L}=\Phi_{i, j, k}, \Phi_{R}=\Phi_{i, j, k+1}$,

$v^{+}=\frac{\max (u, 0) \Delta t}{\Delta x}, \quad v^{-}=\frac{\min (u, 0) \Delta t}{\Delta x}$,

and

$\widehat{\Phi}_{L}=\Phi_{L}+\frac{1}{2}\left(1-v^{+}\right) \Delta z \partial_{z} \Phi_{L}$,

$\widehat{\Phi}_{R}=\Phi_{R}-\frac{1}{2}\left(1+v^{-}\right) \Delta z \partial_{z} \Phi_{R}$.

The flux $F_{y}^{(x)}$ is computed with a corrected level set function $\Phi^{(x)}$ satisfying $f\left(\Phi^{(x)}, \nabla \Phi^{(x)}\right)=\Psi^{(x)}$ and similarly for $F_{z}^{(y)}$. Correction of $\Phi$ is discussed below.

\subsection{Mass redistribution}

Due to numerical errors, values $\Psi>1$ and $\Psi<0$ may occur. We describe our method to correct these overshoots and undershoots without violating mass conservation. Such correction is also used in cells where $0<\Psi<1$ but where no interface is present; such a cell is said to contain numerical vapor. No interface is present in a cell when $\Phi$ in it and in its six surrounding neighbors is of the same sign. We use the following conservative mass redistribution procedure.

1. Determine $\widehat{\Psi}$ by replacing values $\Psi>1$ by $1, \Psi<0$ by 0 and $\Psi \neq 0$ or 1 in cells without an interface by 0 or 1 , whichever is closer.

2. $\epsilon=\hat{\Psi}-\Psi$.

3. Determine $\hat{\epsilon}$ by transport of $\epsilon$ in the direction of the interface in the manner described below.

4. $\Psi=\Psi+\hat{\epsilon}$

5. Determine $\Phi$ from $\Psi$ in the manner described in the next Section.

6. If $\|\hat{\epsilon}\|$ too large, go back to step 1 .

In practice we observe that $\Psi$ needs to be corrected in only a small number of cells (typically $<10$ on a $64^{3}$ grid) and that one iteration of the above procedure suffices.

Transport of $\epsilon$ takes place by solving the following artificial transport equation

$\frac{\partial \epsilon}{\partial \tau}+\nabla \cdot(\mathbf{q} \epsilon)=0$,

until steady state is reached in artificial time $\tau$. Time-stepping is terminated if

$\left\|\frac{\partial \epsilon}{\partial \tau}\right\|_{\infty} \leq$ tol $\vee n_{\epsilon}=0 \vee n>n_{\max }$, where $n_{\epsilon}$ is the number of cells with $|\epsilon|>$ tol and $n$ is the number of time steps. Usually termination occurs for $n<5$. The first order upwind scheme is used. The artificial velocity field $\mathbf{q}=(u, v, w)$ is directed towards the interface, and is chosen as follows:

$u_{i+\frac{1}{2}}=-\operatorname{sign}\left(\left|\Phi_{i+1}\right|-\left|\Phi_{i}\right|\right)$,

and similarly for $v$ and $w$.

\subsection{Time advancement of level set function}

First, a time step is performed with Eq. (18). Let the resulting approximation to $\Phi^{n+3 / 2}$ be denoted by $\Phi^{0}$. This needs to be re-initialized to a distance function, in order to facilitate restriction of regularization of interface forces and smoothing of viscosity to a thin layer around the interface. The re-initialization method of [25] works as follows: we solve numerically until steady state

$$
\begin{aligned}
& \frac{\partial \Phi}{\partial \tau}=N\left(\Phi, \Phi^{0}\right) \equiv \operatorname{sign}\left(\Phi^{0}\right)(1-\|\nabla \Phi\|), \\
& \Phi(\mathbf{x}, 0)=\Phi^{0}(\mathbf{x}),
\end{aligned}
$$

where $\tau$ is an artificial time. Unfortunately, this reinitialization can make small bubbles disappear, and can cause the mass-correction to $\Phi$ (to be described hereafter) be large. This is illustrated by the following example.

Consider the domain $\Omega=(-1 / 2,1 / 2)^{2}$, with interfaces the straight line $x=1 / 4$ and a circle at the origin with radius 2 mesh widths. The initial $\Phi^{0}$ is the distance function represented in Fig. 2c. Numerical re-initialization results in $\Phi$ represented in Fig. 2. The difference between $\Phi$ and $\Phi^{0}$ is significant, and the small circle has disappeared. Correction for mass-conservation results in the level set function presented in Fig. 2a. Comparison with Fig. 2c shows large corrections and a very nonsmooth $\Phi$, which are undesirable features, undermining our mass-conservation strategy.

We therefore propose to change the re-initialization method. The idea is to leave $\Phi$ largely untouched near the interface, exploiting the fact that there $\Phi^{0}$ is already almost a distance function. To make this possible, slight changes in interface position are allowed, keeping in mind that the mass correction that is to follow will move the interface slightly anyway. Let the discretization of Eq. (35) be

$\Phi^{k+1}-\Phi^{k}=\Delta \tau N_{h}\left(\Phi^{k}, \Phi^{0}\right)$.

We change this to

$$
\begin{aligned}
& \Phi^{k+1}-\Phi^{k} \\
& =\Delta \tau N_{h}\left(\Phi^{k}, \Phi^{0}\right)\left(1-q\left(\Phi^{0}\right)\right)+\left(\Phi^{0}-\Phi^{k}\right) q\left(\Phi^{0}\right),
\end{aligned}
$$




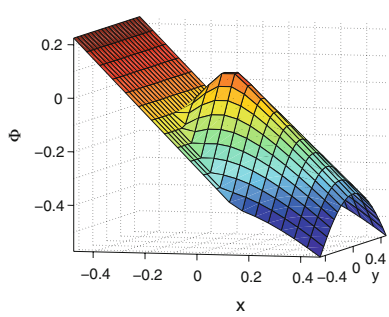

(a) Initial Level-Set function

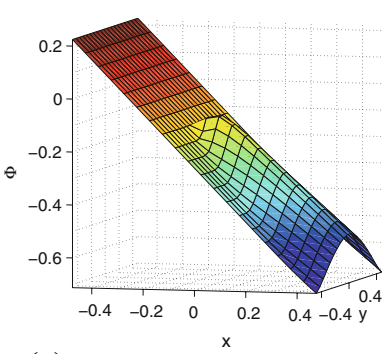

(c) Level-Set function after reinitialization

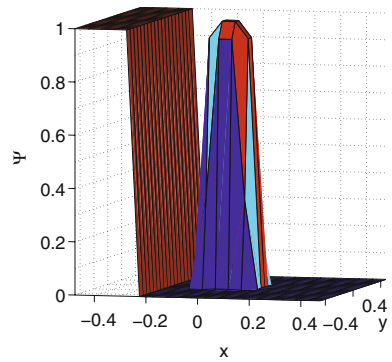

(b) Corresponding Volume-ofFluid function

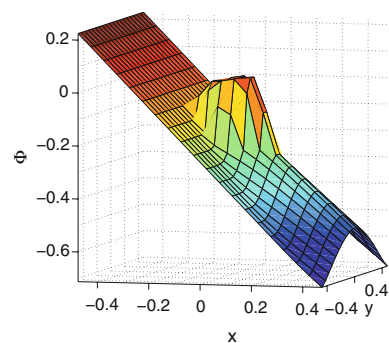

(d) Corrected Level-Set function
Fig. 2 Effect of re-initialization on small bubble

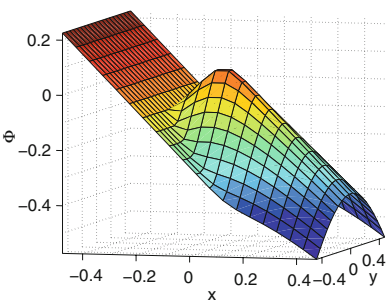

(a) Level-Set function after modified re-initialization

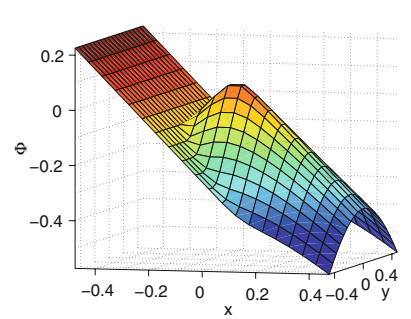

(b) corrected Level-Set function
Fig. 3 Effect of modified re-initialization on small bubble

where

$q\left(\Phi^{0}\right)=\exp \left(-\left(\Phi^{0} / \alpha\right)^{2}\right)$

and

$\alpha=\left(\frac{2}{3}\left(\Delta x^{2}+\Delta y^{2}+\Delta z^{2}\right)\right)^{1 / 2}$

Results for the preceding example are shown in Fig. 3.

Both the re-initialized and the corrected level set function $\Phi$ resemble the initial $\Phi^{0}$ of Fig. 2a, which was our goal.The need for modified re-initialization will be demonstrated in Sect. 5 .

\subsection{Correction of level set function for mass conservation}

Having obtained an acceptable VOF function $\Psi^{n+3 / 2}$, we compute a level set function $\Phi^{n+3 / 2}$ satisfying
$f\left(\Phi_{k}^{n+3 / 2}, \nabla \Phi_{k}^{n+3 / 2}\right)=\Psi^{n+3 / 2}$

(with $f$ given by Eq. (26)) in the following way. Compute an approximation $\Phi^{*}$ to $\Phi^{n+3 / 2}$ by executing a time step with Eq. (18). Next, $\Phi^{*}$ is corrected iteratively by solving in each cell $k$ :

$f\left(\Phi_{k}^{(l+1)}, \nabla \Phi_{k}^{(l)}\right)=\Psi^{n+3 / 2}, \quad \nabla \Phi_{k}^{(1)}=\Phi_{k}^{*}$

When converged sufficiently, we put $\left(\Phi_{k}^{n+3 / 2)}=\Phi_{k}^{(l+1)}\right.$. Equation (41) is solved by Newton iteration. This procedure makes the level set method mass conserving.

\section{Surface tension}

To model surface tension, the continuous surface force/stress (CSF) method [2] is adopted. The interface forces are approximated by a body force $\mathbf{f}$ given by Eq. (7). The curvature $\kappa$ is defined by

$$
\begin{aligned}
\kappa & =\nabla \cdot \frac{\nabla \Phi}{|\nabla \Phi|} \\
& =\frac{\nabla \cdot \nabla \Phi}{|\nabla \Phi|}-\frac{1}{|\nabla \Phi|^{3}} \sum_{i} \sum_{j} \frac{\partial \Phi}{\partial x_{i}} \frac{\partial \Phi}{\partial x_{j}} \frac{\partial^{2} \Phi}{\partial x_{i} \partial x_{j}} .
\end{aligned}
$$

The second order derivatives are approximated by central differences. To avoid difficulties when $|\nabla \Phi|$ vanishes, first order derivatives are approximated by central of one-sided forward or backward differences, whichever has the largest modulus. Because second derivatives are required, $\Phi$ needs to be smooth. When this is not the case for numerical reasons, parasitic currents can occur; these have been studied in $[5,11,17,26]$. With the pure level set method $\kappa$ is usually accurately obtained, but the local mass corrections of the present (MCLS) method makes $\Phi$ nonsmooth, causing local $\mathcal{O}\left(\epsilon / h^{2}\right)$ errors in $\kappa$, where $\epsilon$ is the magnitude of the corrections. We may have $\epsilon=\mathcal{O}(h)$, so that parasitic currents may grow with mesh refinement.

This undesirable situation is illustrated with the so-called Laplace test case, frequently used to study parasitic currents $[11,20,26]$. A spherical bubble with radius $\frac{1}{4} m$ is placed in the center of a cubic domain with dimensions $1 \times 1 \times 1 \mathrm{~m}$. The parameters are $g=0, \sigma=0.01 \mathrm{~kg} / \mathrm{s}^{2}$, and the two fluids have the same density $\rho=1 \mathrm{~kg} / \mathrm{m}^{3}$ and viscosity $\mu=0.1 \mathrm{~kg} / \mathrm{ms}$. The exact solution is flow at rest, so a nonzero velocity is parasitic. In Fig. 4 the time evolution of parasitic currents are shown for three different grid sizes: $32^{3}$, $64^{3}$ and $96^{3}$. We see that $\|\mathbf{u}\|_{\infty}=\mathcal{O}(1 / h)$, and that parasitic currents grow in time.

To suppress parasitic currents, we will re-initialize the level set function only when the interface has travelled some 


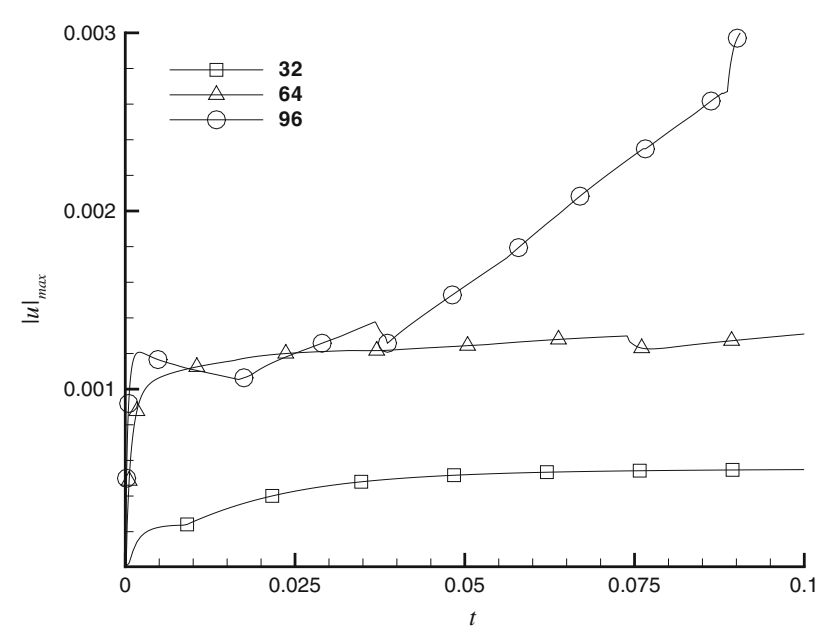

(a) Time evolution

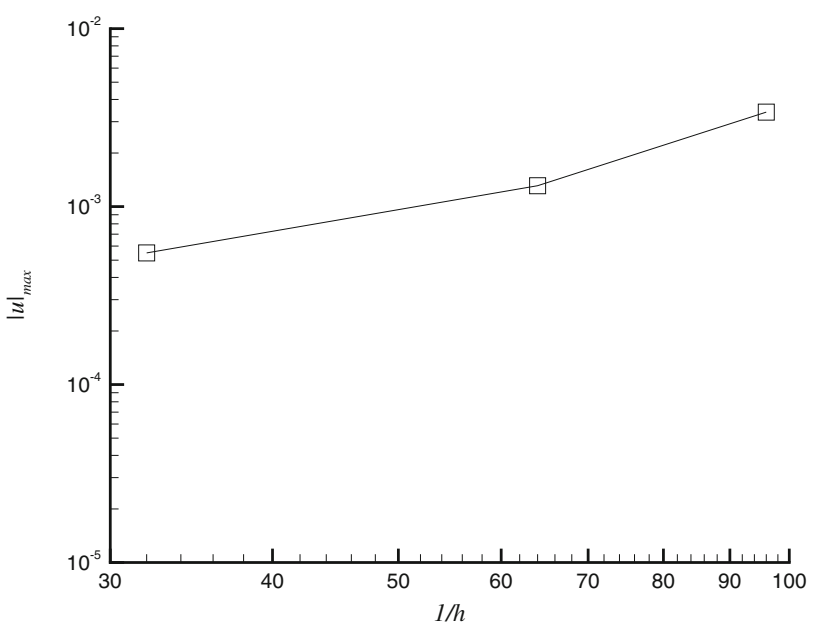

(b) Grid dependence at $t=0.1$

Fig. 4 Parasitic currents for the Laplace test case with three different grids

distance, and we will smooth the curvature $\kappa$. An estimate of the time required for the interface to travel some distance is

$\Delta t_{\text {reinit }}=\frac{\sigma}{\frac{\|u\|_{\infty}}{\Delta x}+\frac{\|v\|_{\infty}}{\Delta y}+\frac{\|w\|_{\infty}}{\Delta z}}$,

where we choose $\sigma=0.1$. Re-initialization is only performed at time intervals $\Delta t_{\text {reinit }}$. For curvature smoothing (common in VOF [17,20] and front-tracking methods [4]) we proceed as follows. The following properties are desirable:

1. The curvature is smooth,

2. The curvature is the divergence of $\frac{\nabla \Phi}{|\nabla \Phi|}$,

3. When little smoothing is required, the curvature has to correspond to the interface $\Phi=0$.
To meet these demands, we let $\kappa$ satisfy

$\frac{\partial \kappa}{\partial \tau}=\nabla \cdot d \nabla \kappa$

where $\tau \in\left(0, h^{2} / 6\right)$ is a pseudo-time variable. The first two requirements are satisfied if $d$ is smooth. We choose $d$ as follows:

$d=1-\exp \left(\frac{-\Phi^{2}}{\Delta x^{2}+\Delta y^{2}+\Delta z^{2}}\right)$.

With this choice of $d$, the last demand is also met, because near the interface $d \approx 0$, unless $\kappa$ is nonsmooth. Explicit Euler time stepping with $\Delta \tau=N / 16$ is used. The need for curvature smoothing and, in particular, the diffusion coefficient as prescribed by Eq. (45), will be demonstrated in Sect. 5.

Results for the Laplace test-case are shown in Fig. 5.

The circle symbols in Fig. 5b show that the parasitic currents converge with $\mathcal{O}(h)$.

\section{Applications}

The three-dimensional mass-conserving level set (MCLS) method described above is used to compute the flow induced by a rising air bubble in water.

The parameters are

$$
\begin{aligned}
g & =9.8 \frac{\mathrm{m}}{\mathrm{s}^{2}}, & \sigma & =0.0728 \frac{\mathrm{kg}}{\mathrm{s}^{2}}, \\
\rho_{w} & =10^{3} \frac{\mathrm{kg}}{\mathrm{m}^{3}}, & \rho_{a} & =1.226 \frac{\mathrm{kg}}{\mathrm{m}^{3}}, \\
\mu_{w} & =1.137 \times 10^{-3} \frac{\mathrm{kg}}{\mathrm{ms}}, & \mu_{a} & =1.78 \times 10^{-5} \frac{\mathrm{kg}}{\mathrm{ms}} .
\end{aligned}
$$

The domain is a cube with edge length $0.01 \mathrm{~m}$. Initially, the flow is at rest, with a flat free surface at height $z_{1}=0.0075 \mathrm{~m}$ and a spherical bubble with radius $0.00125 \mathrm{~m}$ and center at $z_{0}=0.0025 \mathrm{~m}$ on the vertical axis of the cube. Results with zero surface tension on a $96 \times 96 \times 96$ grid are shown in Fig. 6. The snapshots are taken at time intervals of $0.005 \mathrm{~s}$. The bubble deforms and breaks up to form a bell- and ringlike structure, just before it breaks through the free surface. In [22] similar computations are presented with low surface tension. There is good agreement between these results and the snapshots presented in Fig. 7. Note that we have chosen different viewpoints for each snapshot to mach the ones in [22].

In Fig. 12 the rise speed $w_{c}$ of the droplet before collision with the free surface is plotted as function of time $t$.

The rise speed is in good agreement for both grids. The rise speed reaches a maximum just after the bubble starts 


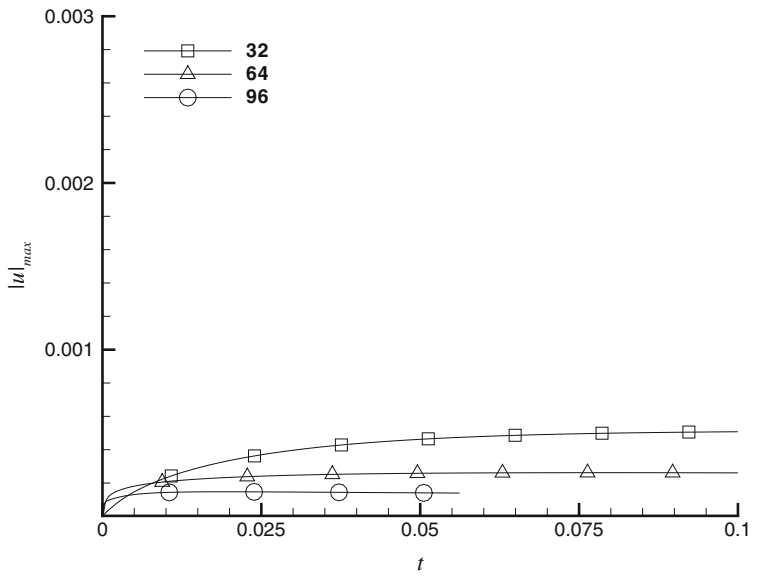

(a) The parasitic currents are bounded in time for all grids

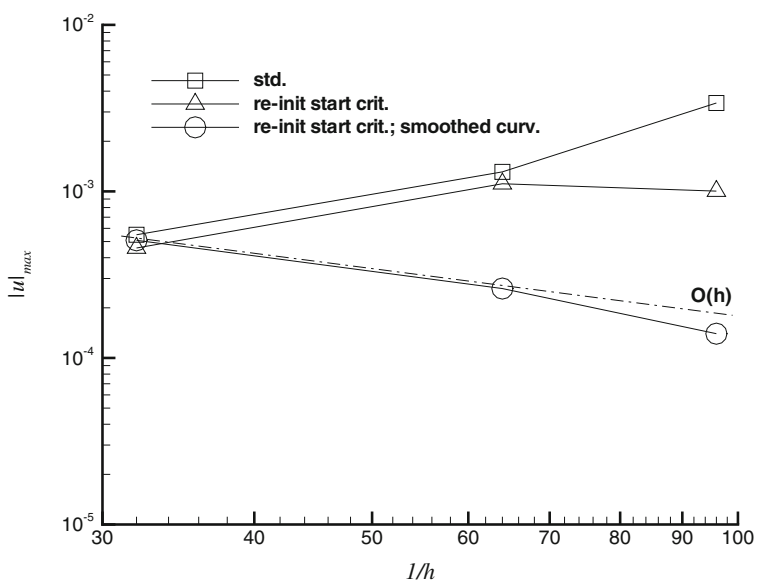

(b) Grid dependence at $t=0.1$; Note that the two previous cases ('std' and 're-init start crit.') are also included in this figure; The parasitic currents decrease further due to smoothing of the curvature

Fig. 5 Parasitic currents for the Laplace test case with three different grids; time-step criterion in re-initialization and smoothed curvature

moving. A local maximum is reached again before impact with the free surface in top of the domain.

The shape of the bubble just before it breaks through the free surface is shown in Fig. 8.

The bubble has become a spherical cap with radius $R_{S}=$ $0.0018 \mathrm{~m}$. In [1] the following expression for the rise velocity of a spherical cap without surface tension, based on potential flow, is derived:

$w_{s}=\frac{2}{3} \sqrt{g R_{s}}=0.0885 \mathrm{~m} / \mathrm{s}$.

This speed is indicated in Fig. 12. Before reaching the surface and after more or less getting spherical cap shape, the bubble's rise velocity shows satisfactory agreement with spherical cap theory.
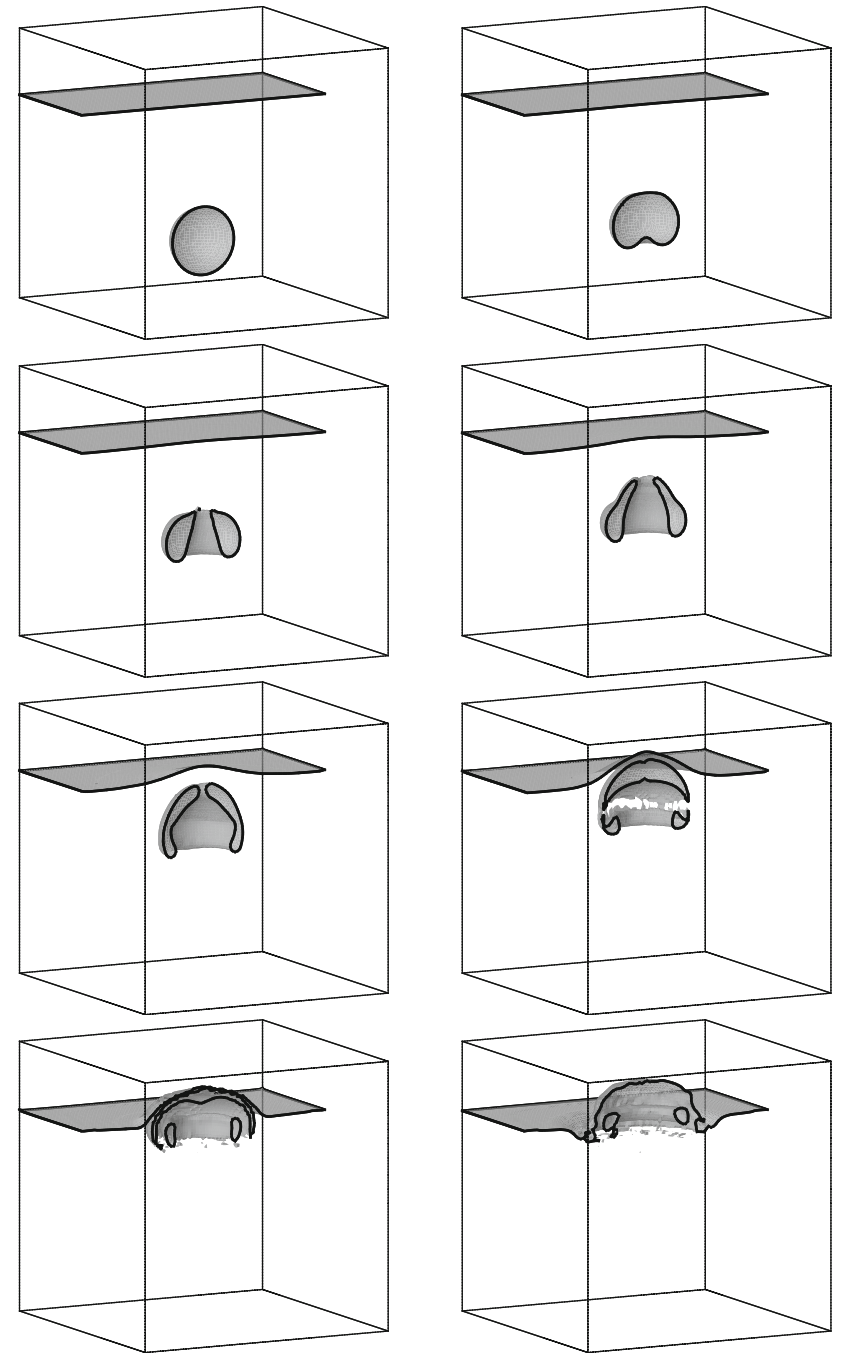

Fig. 6 Rising bubble without surface tension; $96^{3}$ grid

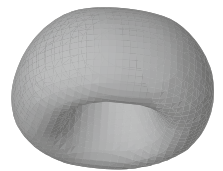

(a) $t=0.009 \mathrm{~s}$

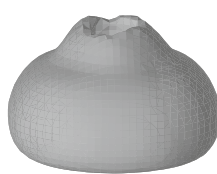

(c) $t=0.013 \mathrm{~s}$

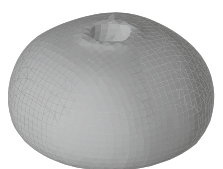

(b) $t=0.01 \mathrm{~s}$

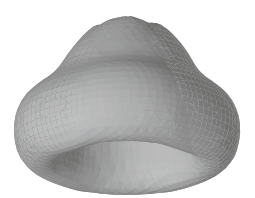

(d) $t=0.015 \mathrm{~s}$
Fig. 7 Rising bubble without surface tension; $96^{3}$ grid; zoomed in. Note that we have chosen different viewpoints for each snapshot to mach the ones in [22]

The effect of grid resolution can be seen from Fig. 9, where results for the coarser $64^{3}$ grid are shown. Good agreement for the two grids utilized is observed. 


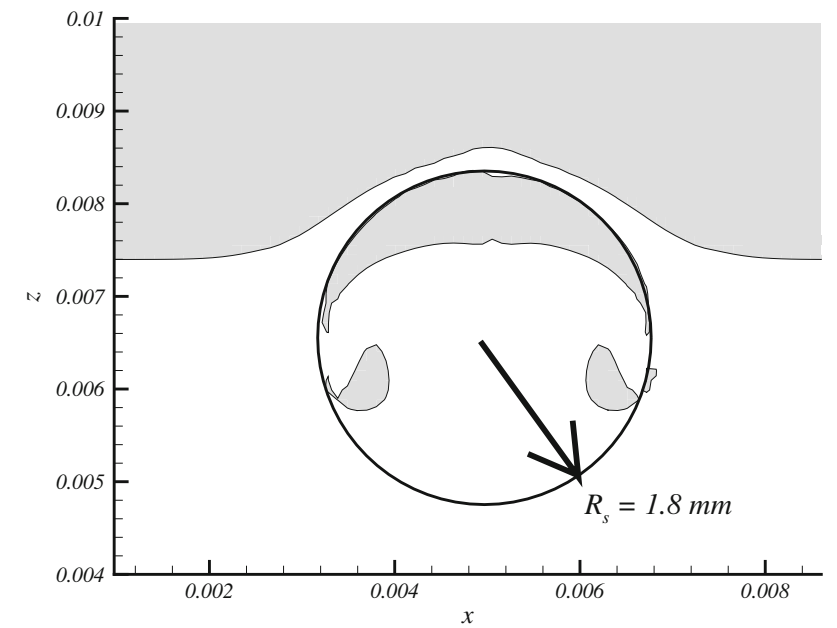

Fig. 8 Bubble without surface tension just before it breaks through the free surface; symmetry plane cross-section; $R_{S}$ is the radius of the spherical cap
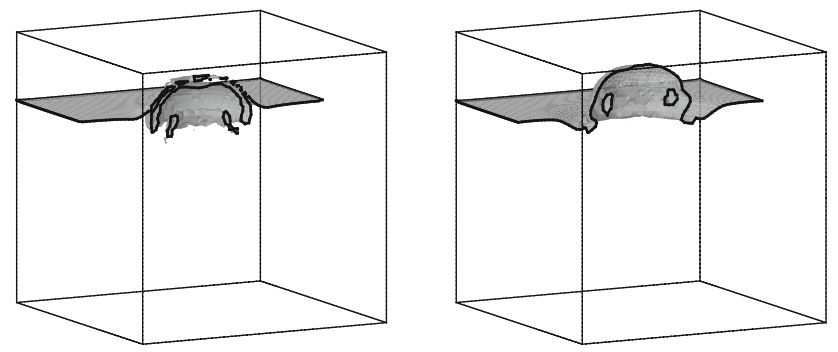

Fig. 9 Bubble when it breaks through the free surface; $64^{3}$ grid

In Fig. 10 results with surface tension are presented. The deformation of the bubble is significantly reduced.

The shape of the jet that appears after the bubble broke through the free surface is shown in Fig. 11 for the coarser $64^{3}$ grid.

There is good agreement with the finer mesh.

In Fig. 12 the rise speed $w_{c}$ of the bubble with surface tension is plotted for both grids. The agreement between both grids is good. When the bubble is just released, the acceleration of the bubble with surface tension is lower than the bubble without surface tension. But instead of reaching a maximum rise velocity, the bubble with surface tension continues to accelerate, reaching higher speeds than without surface tension. A peak is observed just before collision with the free surface in top of the domain. This suggests that the bubble is sucked towards the free-surface before merging with it.

Next, a falling water droplet in air is considered. The parameters and the geometry are the same as for the rising bubble. But the droplet is released at half the height of the domain, i.e. $z_{0}=0.005 \mathrm{~m}$; and the free surface is initially located at $z_{1}=0.0025 \mathrm{~m}$. The initial shape of the droplet is spherical with radius $0.00125 \mathrm{~m}$.
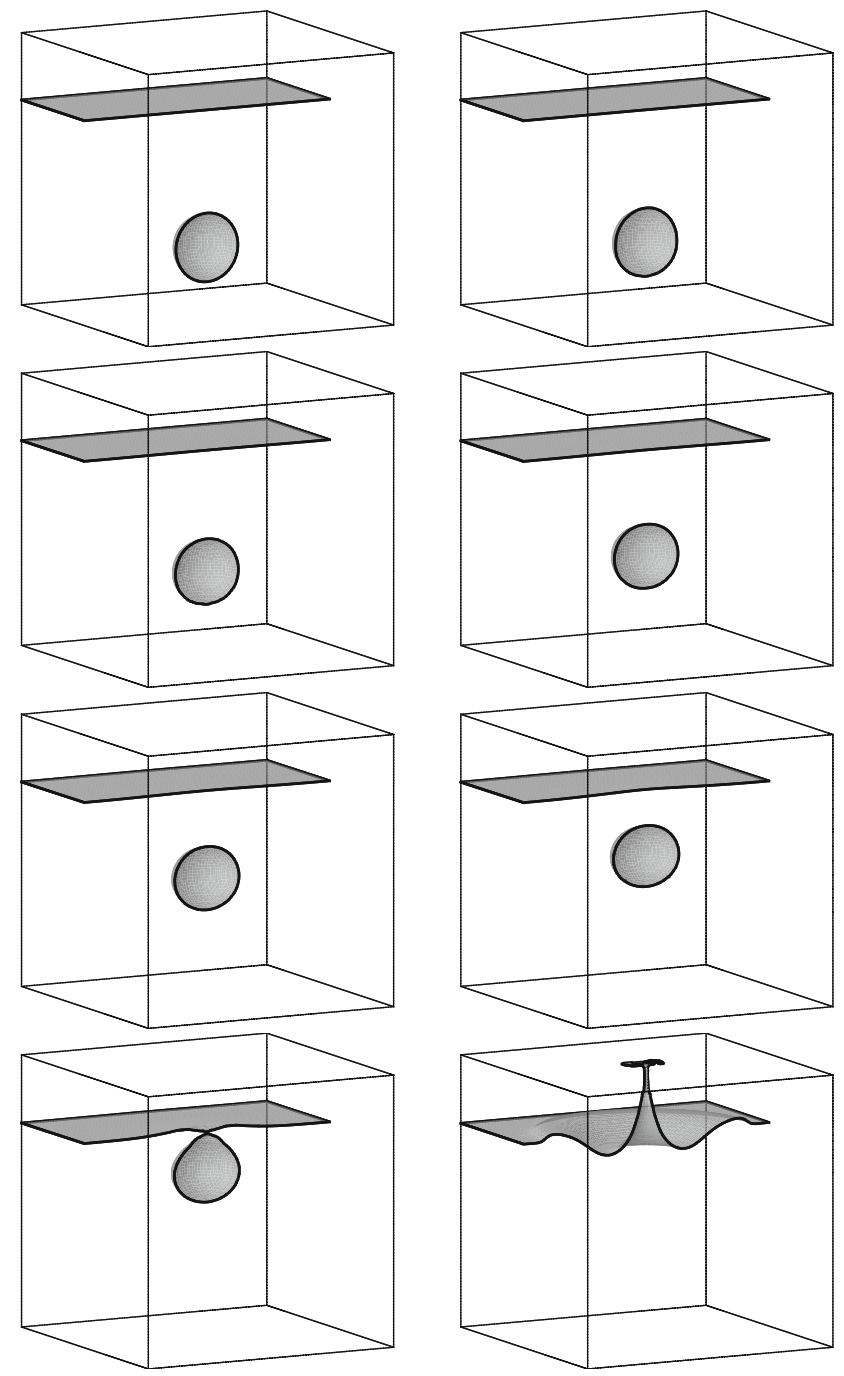

Fig. 10 Rising bubble with surface tension; $96^{3}$ grid
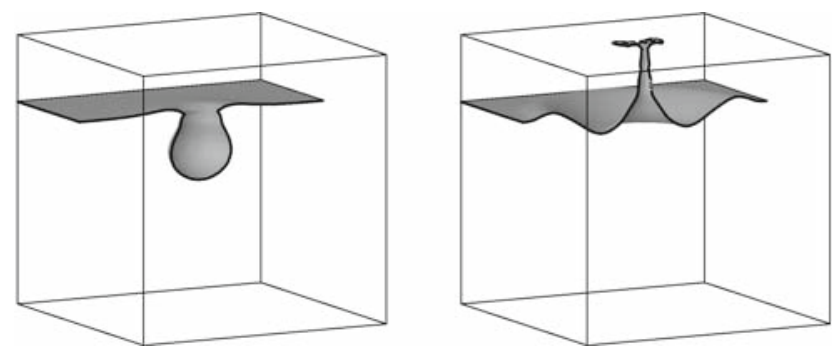

Fig. 11 Just before and right after the bubble breaks through the free surface; $64^{3}$ grid

Results are shown in Fig. 13. The snapshots are taken at intervals of $0.01 \mathrm{sec}$. The droplet accelerates after it is released and hits the free surface. A jet appears after collision that reaches up to the ceiling of the domain.

The jet after collision of the droplet with the free surface for the $64^{3}$ grid is presented in Fig. 14.

The jet shows similar behavior for the two grids utilized. 


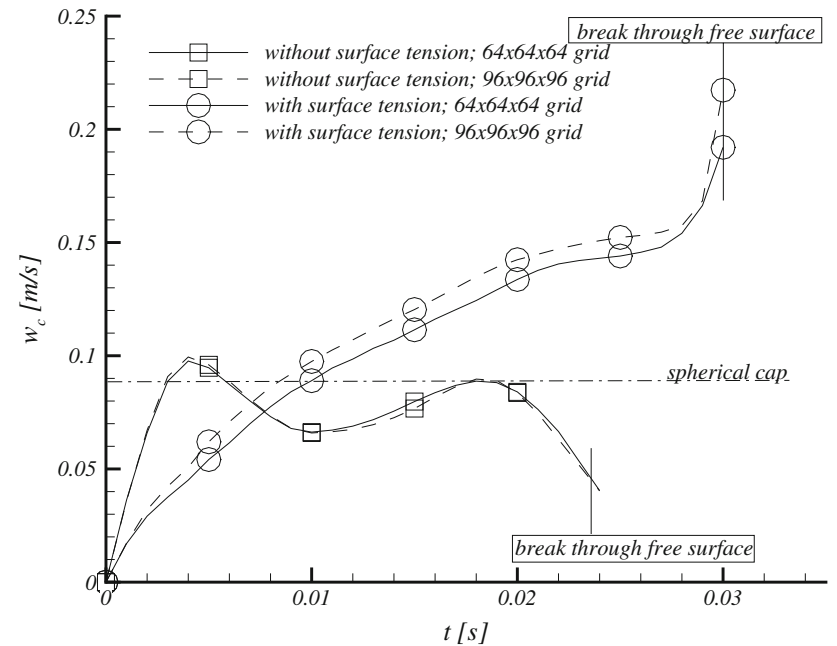

Fig. 12 Rise speed of the bubble with surface tension; marks are at equally spaced time intervals of $0.005 \mathrm{~s}$

In Fig. 15 the fall velocity $w_{c}$ of the droplet until impact with the free surface is plotted as function of time. Good agreement of the fall velocity with the free-fall velocity-gt is observed. Next, surface tension is included. This restricts deformation of the droplet so that a greater release height (now chosen as $z_{0}=0.0075 \mathrm{~m}$ ) is possible. Without surface tension break-up before impact encumbers computation. In Fig. 16 results are presented. Although the release height of the droplet is larger than without surface tension, the jet after collision is considerably smaller. This is the effect of surface tension that tries to minimize the interfacial area.

The effect of grid resolution just after collision with the free surface is shown in Fig. 17. Similar results for the two grids are observed.

In Fig. 18 the fall velocities are compared with the free fall. Both grids give the same fall velocity, which is larger than free-fall, which is unphysical. This is believed to be caused by the CSF approach, because the computation without surface tension gives a correct result. The total force acting on a bubble or droplet due to surface tension should vanish, since the surface tension forces are internal forces and the interface is closed. However; with the CSF approach a non-conservative expression is obtained. This means that numerically the forces do not necessarily add up to zero. Inspection of the numerical data shows that

$\sum_{k} \frac{1}{\rho} \mathbf{f}_{s} \approx-10^{-8}$

The acceleration of the droplet will therefore be

$g+\frac{1}{\frac{4}{3} \pi R^{3}} \sum_{k} \frac{1}{\rho} \mathbf{f}_{s} \approx-11 \mathrm{~m} / \mathrm{s}^{2}$,
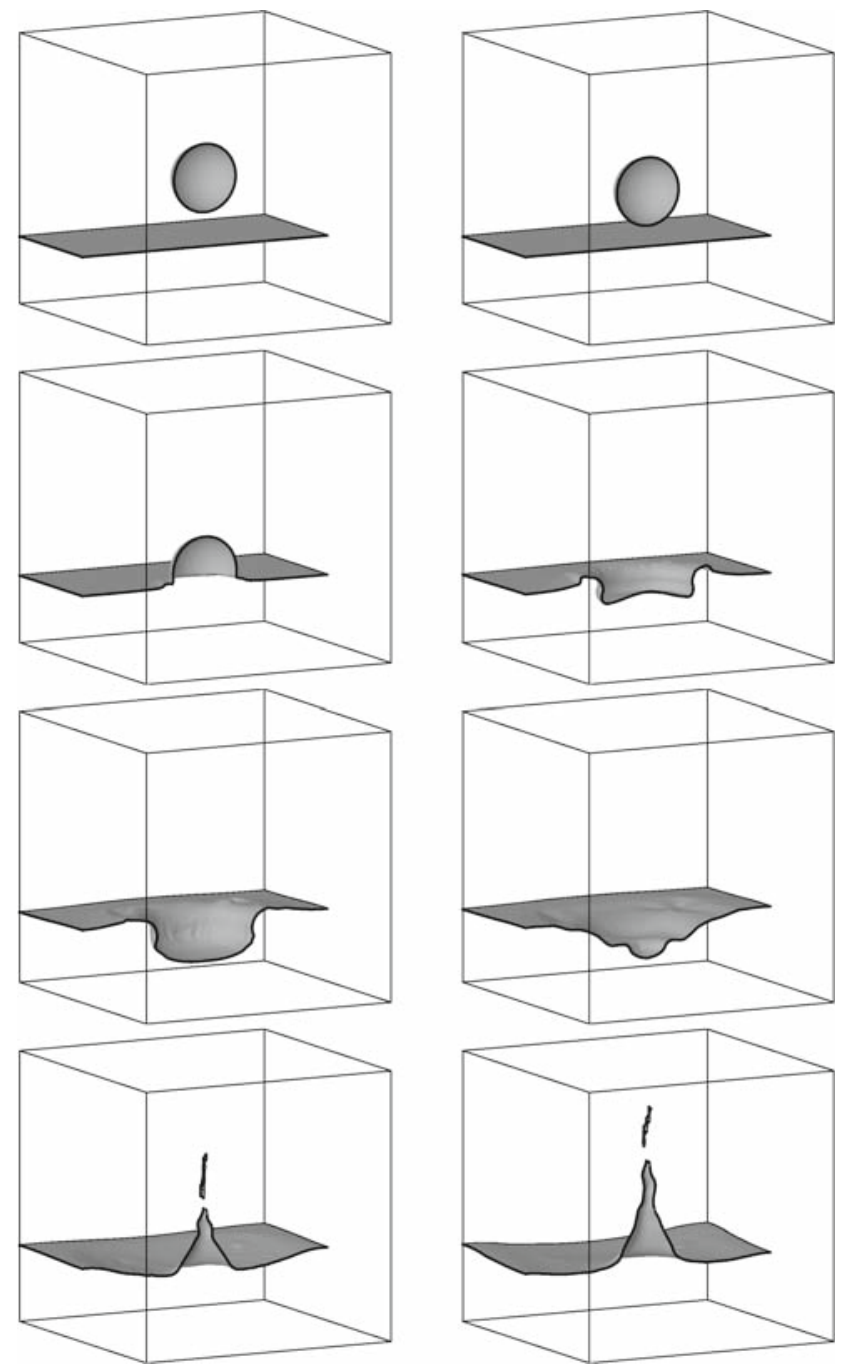

Fig. 13 Falling droplet without surface tension; $96^{3}$ grid
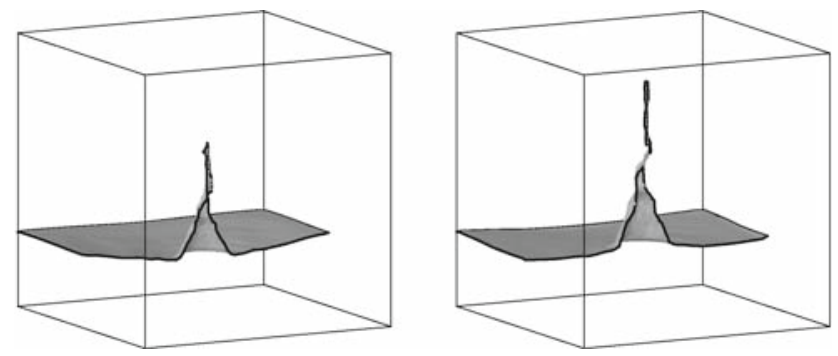

Fig. 14 The jet just after the droplet collided with the free surface; $64^{3}$ grid

This is in agreement with Fig. 18. Note that a conservative formulation of the surface tension forces prevents such difficulties rigorously and would be preferred.

Let us now have a detailed look into the effects of the various components of the MCLS method that have been described in this paper. Firstly, the effects of mass conservation are illustrated by consider the following cases: 


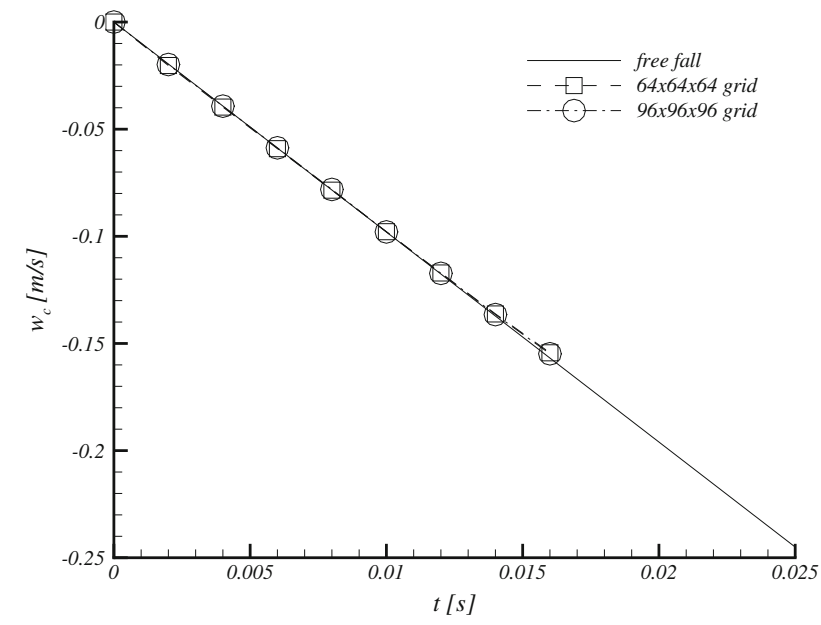

Fig. 15 Fall velocity of droplet without surface tension compared with free-fall
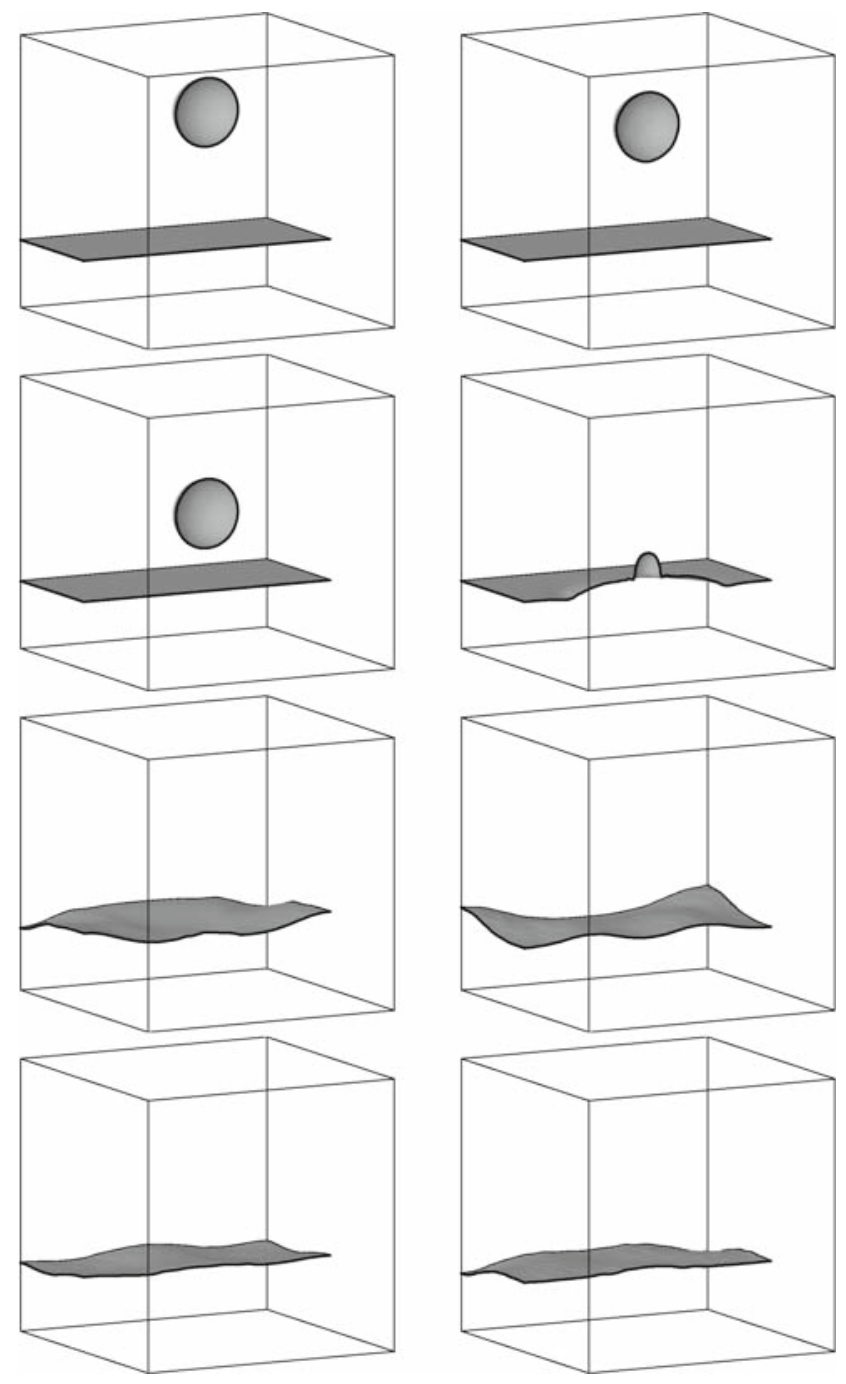

Fig. 16 Falling droplet with surface tension; $96^{3}$ grid

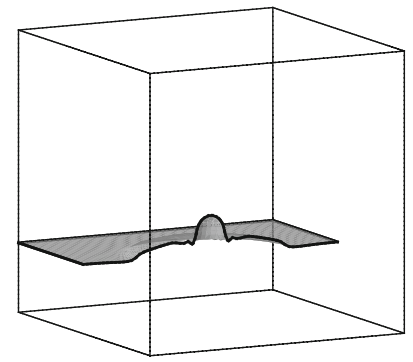

Fig. 17 The droplet just after collision; $64^{3}$ grid

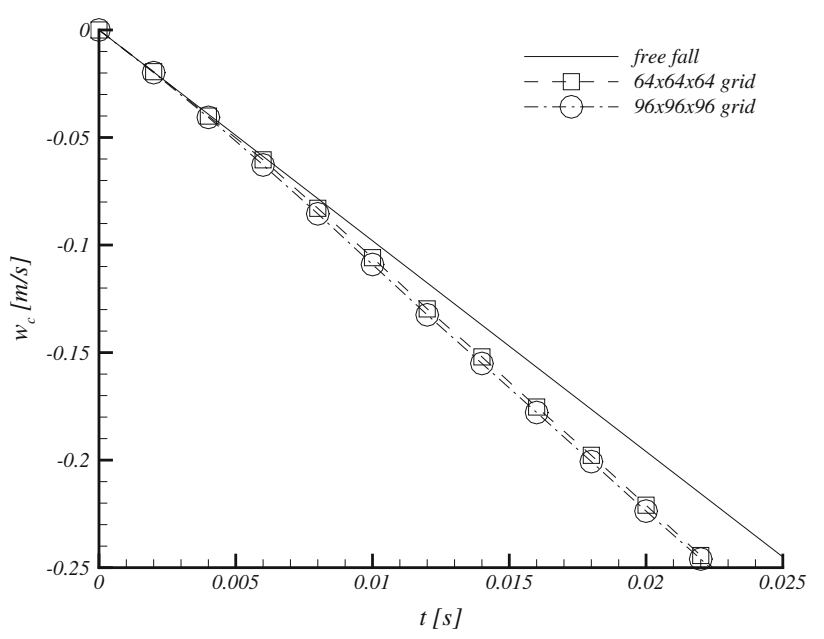

Fig. 18 Fall speed of the droplet with surface tension compared with the free-fall velocity

- pure level set method, with the standard re-initialization, i.e. Eqs. (18), (35),

- pure level set method, with the modified re-initialization as described in Sect. 3.4, i.e. Eq. (37),

- MCLS, without the mass redistribution procedure of Sect. 3.3,

- MCLS, with mass redistribution,

where we choose a tolerance of $10^{-4}$ for the error in the volume-of-fluid function. The relative mass errors of the rising bubble are plotted in Fig. 19. For pure level set advection with standard re-initialization, the bubble looses its mass until it has completely disappeared at $t \approx 0.009 \mathrm{~s}$. An improvement is observed by the modified re-initialization. Still, mass errors are about 10 percent at $t=0.025 \mathrm{~s}$. This error is reduced by an order of magnitude by the MCLS approach and even further when the mass errors are redistributed according to Sect. 3.3.

Next, the effect of curvature smoothing is illustrated by considering:

- no curvature smoothing,

- standard curvature smoothing, i.e. $d=1$ in Eq. (44),

- curvature smoothing with $d$ prescribed by Eq. (45). 


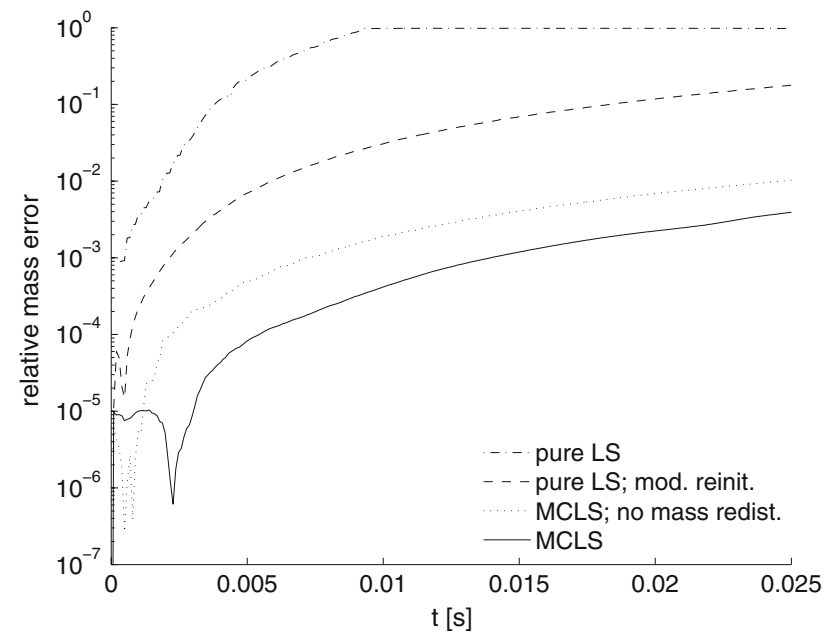

Fig. 19 Relative mass errors for the rising bubble case with surface tension; $96^{3}$ grid

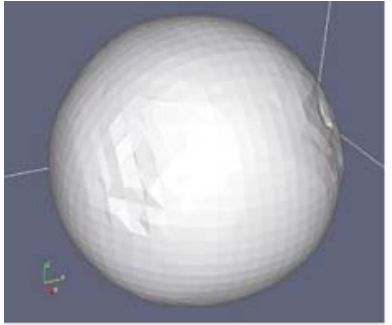

(a) No curvature smoothing

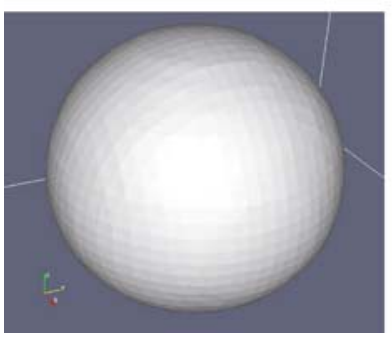

(c) Proper curvature smoothing

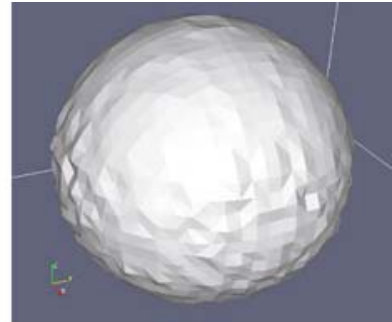

(b) Standard curvature smoothing

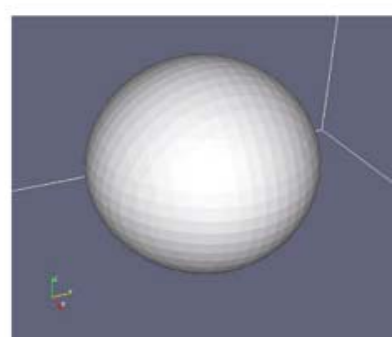

(d) Pure level set; unmodified reinitialization
Fig. 20 Effects of curvature smoothing on the rising bubble; $t=0.007 \mathrm{~s} ; 96^{3}$ grid

The shape of the bubble at $t=0.008 \mathrm{sec}$ is depicted in Fig. 20. Without curvature smoothing, the interface gets distorted locally, as can be seen from Fig. 20a. In fact, the computations will break down just after this moment due to the high (parasitic) currents. When the curvature is smoothed in a straightforward fashion, by taking $d=1$ in Eq. (44), no such difficulties are encountered. However, the interface is highly distorted, or wrinkled, as can be seen from Fig. $20 \mathrm{~b}$. This clearly demonstrates the drawback of straightforward curvature smoothing: although parasitic currents are suppressed, small perturbations, or wrinkles, on the interface are no longer dampened by surface tension, since the corresponding perturbed curvatures are now smoothed. Or, to put it the other way around, for a given curvature distribution, there exists more than one possible interface position due to curvature smoothing.

Finally, no wrinkles are observed once the diffusion coefficient $d$ in Eq. (44) is as in Eq. (45). This is shown in Fig. 20. Since $d$ vanishes for $\Phi=0$, small perturbations of the interface will still induce stabilizing surface tension forces. Since $d$ is smooth and non-zero away from the interface, parasitic currents are suppressed as was demonstrated in Sect. 4. The interface is equally smooth compared to pure level set advection, see Fig. 20d. However, note the significant difference in mass conservation.

We now turn to comparison with experimental data for the rising bubble case. In [8] computations are made with a front tracking and a volume-of-fluid method, and comparison with experiment is made. The flow is characterized by the Eötvos and Morton numbers:

$\mathrm{E} \ddot{0}=\frac{g\left(\rho_{1}-\rho_{0}\right)(2 R)^{2}}{\sigma}, \quad$ Mo $=\frac{g \mu_{1}^{4}\left(\rho_{1}-\rho_{0}\right)}{\rho_{1}^{2} \sigma^{3}}$.

where subscripts 1 and 2 refer to the heavier fluid outside and the lighter fluid inside the bubble, respectively. The Reynolds number based on the final rise velocity $w_{c}$ is defined as

$R e=\frac{\rho_{l} w_{c} 2 R}{\mu_{1}}$

When the flow is fully developed, the drag force $F_{d}$ is balanced by the buoyancy force, so that

$F_{d}=\frac{4}{3} \pi R^{3} g\left(\rho_{1}-\rho_{0}\right)$.

The drag coefficient is defined by

$c_{d}=\frac{F_{d}}{\frac{1}{2} \rho_{1} w_{c}^{2} \pi R^{2}}$.

In [8] the following three relations to represent experimental results are given:

(I) $c_{d}=14.9 R e^{-0.78}$,

(II) $c_{d}=\frac{3.05\left(783 \gamma^{2}+2142 \gamma+1080\right)}{(60+29 \gamma)(4+3 \gamma)} R e^{-0.74}$,

(III) $c_{d}=\max \left(\min \left(\frac{A}{R e_{b}\left(1+0.15 R e_{b}^{0.6882}\right)}, \frac{3 A}{R e_{b}}\right)\right.$,

$$
\left.\frac{8}{3} \frac{\mathrm{EO}}{\mathrm{EO}+4}\right) \text {, }
$$

where $\gamma=\rho_{0} / \rho_{1}$. The resulting Reynolds numbers are shown in Table 1. Compared to experiment, the computations of [8] are about $40 \%$ too high for the VOF method and $10 \%$ 
Table 1 Reynolds numbers of the rising bubble compared with the numerical results from Gunsing [8] and experimental data; Eö $=1.0$; $\log (M)=-3.8$

\begin{tabular}{llll}
\hline & & & $R e$ \\
\hline Gunsing & {$[8]$} & VOF & 8.2 \\
Gunsing & {$[8]$} & front tracking & 6.4 \\
Experimental & (I) & Eq. (53) & 5.2 \\
Experimental & (II) & Eq. (54) & 5.3 \\
Experimental & (III) & Eq. (55) & 5.8 \\
This work & & & 4.6 \\
\hline
\end{tabular}

for the front tracking method. Our work under-predicts the rise velocity with about $10 \%$.

Next, we turn to multi-bubble computations. The coalescence of two rising bubbles is studied in [4]. Two bubbles are initially at rest and aligned on the center line of the computational domain with dimensions $L_{x}=L_{y}=0.01 \mathrm{~m}$, $L_{z}=0.02 \mathrm{~m}$. The radii of the bubbles are $R=0.0013 \mathrm{~m}$. The distance from the bottom bubble to the floor of the domain is $z_{0}=0.0025 \mathrm{~m}$. The distance between the bubbles is
$\Delta z=0.0055 \mathrm{~m}$. This makes the gap between the bubbles $\Delta z-2 R=0.0004 \mathrm{~m}$. The parameters are:

$$
\begin{aligned}
g & =9.8 \mathrm{~m} / \mathrm{s}^{2}, & \sigma & =5.8 \times 10^{-4} \mathrm{~kg} / \mathrm{s}^{2}, \\
\rho_{0} & =440 \mathrm{~kg} / \mathrm{m}^{3}, & \rho_{1} & =880 \mathrm{~kg} / \mathrm{m}^{3}, \\
\mu_{0} & =0.00625 \mathrm{~kg} / \mathrm{ms}, & \mu_{1} & =0.00125 \mathrm{~kg} / \mathrm{ms},
\end{aligned}
$$

where subscripts 0 and 1 indicate the lighter and heavier fluid respectively.The coalescence of two rising bubbles is illustrated in Fig. 21. Snapshots are taken at time intervals of $0.02 s$, starting from the initial condition. Good qualitative agreement is found with the results presented in [4]. But our rise velocity is about $35 \%$ larger. Note that rise velocities predicted by our model have been validated in the previous sections.

Next, let the bubbles be misaligned initially by a horizontal distance equal to the radius. Results are plotted in Fig. 22. The bottom bubble is sucked into the top bubble. A thin tail remains after merging, that never completely disappears. These simulations have also been performed in [4]. Again, qualitative agreement is good, but as before the rise velocity is different. Furthermore, the tail after merging of the two
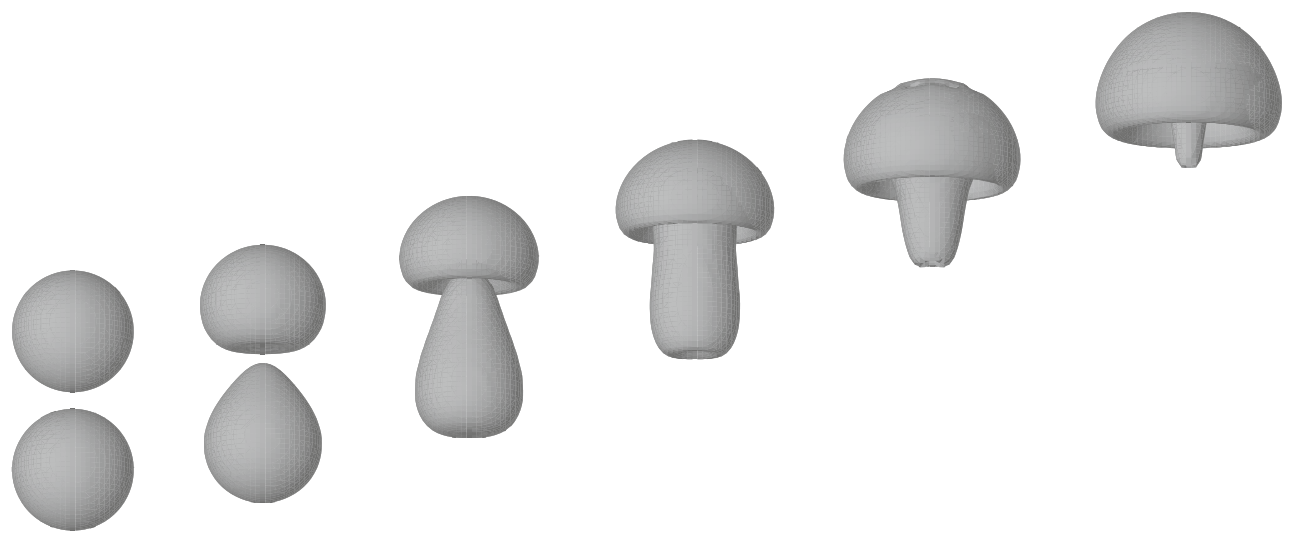

$t=0.02 \mathrm{~s}$

$t=0.04 \mathrm{~s}$

$t=0.06 \mathrm{~s}$

$t=0.08 \mathrm{~s}$

$t=0.1 \mathrm{~s}$

Fig. 21 Coalescence of two aligned rising bubbles; $96 \times 96 \times 192$ grid

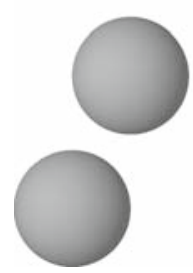

$t=0 s$

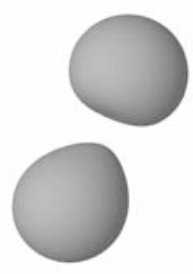

$t=0.02 \mathrm{~s}$

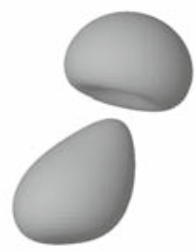

$t=0.04 \mathrm{~s}$
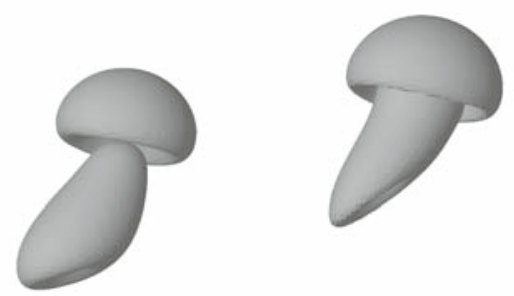

$t=0.06 s$

$t=0.08 s$

$t=0.1 s$

Fig. 22 Coalescence of two misaligned rising bubbles; $96 \times 96 \times 192$ grid 
Table 2 Computational costs measured in CPU seconds per time step for the rising bubble test case on a $96 \times 96 \times 192$ mesh

\begin{tabular}{|c|c|c|c|}
\hline & & MCLS & \\
\hline Level-Set advection & & 0.4 & \\
\hline re-initialization & & 2.3 & \\
\hline \multicolumn{4}{|l|}{ VOF advection } \\
\hline flux $x$ & 0.7 & & \\
\hline correct & 3.9 & & \\
\hline flux $y$ & 0.8 & & \\
\hline correct & 3.9 & & \\
\hline flux $z$ & 0.8 & & \\
\hline correct & 3.9 & & \\
\hline redist & 6.1 & & \\
\hline total & & 20.1 & \\
\hline Total advection & & & 23 \\
\hline curv. smooth. & & & 25 \\
\hline Poisson solver & & & 126 \\
\hline Total time-step & & & 180 \\
\hline
\end{tabular}

bubbles suddenly disappears in [4], whereas according to Fig. 22 the tail does not disappear; it just becomes becomes thinner as time passes. The interface does also seem to be more irregular in [4], notwithstanding application of a socalled "undulation removal" to smooth the interface. Note that in the present work the interface is not smoothed. Nevertheless, a smooth interface is obtained.

To conclude, we give a breakdown of computing time for the last example in Table 2. The numbers correspond to CPU seconds spent per time-step for the misaligned rising bubble test case on a $96 \times 96 \times 192$ mesh. The interface advection takes approximately $13 \%$ of the total time. About $70 \%$ of the time-step is spent on solving the Poisson equation (Eq. 11). This is also generally the case for single phase Navier-Stokes computations, so until the Poisson equation is solved more efficiently, there seems to be no pressing need to make the interface treatment more efficient.

\section{Conclusion}

A mass-conserving level set (MCLS) method for threedimensional two-phase flows, allowing coalescence and break-up of bubbles, has been presented. Difficulties in re-initializing the level set function and in computation of surface tension have been investigated, and remedies were proposed. The method was validated by some suitable test cases, and finally computations involving interaction of two bubbles were described. It was shown that the method can handle very large density ratios, and that the handling of interfaces requires not more than a reasonable part of total computing time.
Open Access This article is distributed under the terms of the Creative Commons Attribution Noncommercial License which permits any noncommercial use, distribution, and reproduction in any medium, provided the original author(s) and source are credited.

\section{References}

1. Batchelor, G.: An Introduction to Fluid Dynamics. Cambridge University Press, Cambridge (1967)

2. Brackbill, J., Kothe, D., Zemach, C.: A continuum method for modeling surface tension. J. Comput. Phys. 100, 335-354 (1992)

3. Chang, Y., Hou, T., Merriman, B., Osher, S.: A level set formulation of Eulerian interface capturing methods for incompressible fluid flows. J. Comput. Phys. 124, 449-464 (1996)

4. de Sousa, F., Mangiavacchi, N., Nonato, L., Castelo, A., Tomé, M., Ferreira, V., Cumanito, J., McKee, S.: A front-tracking/ front-capturing method for the simulation of 3D multi-fluid flows with free surfaces. J. Comput. Phys. 198, 469-499 (2004)

5. Enright D., Nguyen D., Gibou F., Fedkiw R (2003) Using the particle level set method and a second order accurate pressure boundary condition for free surface flows. In: Proceedings of the FEDSM03 3rd ASME/JSME Joint Fluids Engineering Conference

6. Frolković, P., Mikula, K.: High-resolution flux-based level set method. SIAM J. Sci. Comput. 29(2), 579-597 (2007)

7. Groß, S., Reusken, A.: An extended pressure finite element space for two-phase incompressible flows with surface tension. J. Comput. Phys. 224, 40-58 (2007)

8. Gunsing M (2004) Modelling bubbly flows using volume of fluid, front tracking and discrete bubble models. Ph.D. thesis, University of Twente, The Netherlands

9. Harvie, D., Fletcher, D.: A new volume of fluid advection algorithm: the defined donating region scheme. Int. J. Numer. Methods Fluids 35, 151-172 (2001)

10. Kang, M., Fedkiw, R., Liu, X.D.: A boundary condition capturing method for multiphase incompressible flow. J. Sci. Comput. 323-360 (2000)

11. Lafaurie, B., Nardone, C., Scardovelli, R., Zaleski, S., Zanetti, G.: Modelling merging and fragmentation in multiphase flows with SURFER. J. Comput. Phys. 113, 134-147 (1994)

12. Li, Z., Lai, M.C.: The immersed interface method for the NavierStokes equations with singular forces. J. Comput. Phys. 171, 822-842 (2001)

13. Marchandise, E., Geuzaine, P., Chevaugeon, N., Remacle, J.F.: A stabilized finite element method using a discontinuous level set approach for the computation of bubble dynamics. J. Comput. Phys. 225, 949-974 (2007)

14. Mulder, W., Osher, S., Sethian, J.: Computing interface motion in compressible gas dynamics. J. Comput. Phys. 100, 209-228 (1992)

15. Olsson, E., Kreiss, G., Zahedi, S.: A conservative level set method for two phase flows II. J. Comput. Phys. 225, 785-807 (2007)

16. Osher, S., Fedkiw, R.: Level set methods: an overview and some recent results. J. Comput. Phys. 169, 463-502 (2001)

17. R, S., Zaleski, S.: Direct numerical simulation of free-surface and interfacial flow. Ann. Rev. Fluid Mech. 31, 567-603 (1999)

18. Rider, W., Kothe, D.: Reconstructing volume tracking. J. Comput. Phys. 141, 112-152 (1998)

19. Rudman, M.: Volume-tracking methods for interfacial flow calculations. Int. J. Numer. Methods Fluids 24, 671-691 (1997)

20. Rudman, M.: A volume-tracking methods for incompressible multifluid flow with large density variations. Int. J. Numer. Methods Fluids 28, 357-378 (1998)

21. Sussman, M.: A second order coupled level set and volume-of-fluid method for computing growth and collapse of vapor bubbles. J. Comput. Phys. 187, 110-136 (2003) 
22. Sussman, M., Almgren, A., Bell, J., Colella, P., Howell, L., Welcome, M.: An adaptive level set approach for incompressible two-phase flows. J. Comput. Phys. 148, 81-124 (1999)

23. Sussman, M., Fatemi, E., Smereka, P., Osher, S.: An improved level set method for incompressible two-phase flows. Comput. Fluids 27, 663-680 (1998)

24. Sussman, M., Puckett, E.: A coupled level set and volume-of-fluid method for computing 3D and axisymmetric incompressible twophase flows. J. Comput. Phys. 162, 301-337 (2000)

25. Sussman, M., Smereka, P., Osher, S.: A level set approach for computing solutions to incompressible two-phase flow. J. Comput. Phys. 114, 146-159 (1994)

26. Tryggvason, G., Bunner, B., Esmaeeli, A., Juric, D., Al-Rawahi, N., Tauber, W., Han, J., Nas, S., Jan, Y.J.: A front-tracking method for the computation of multiphase flow. J. Comput. Phys. 169, 708759 (2001)

27. van der Pijl, S.P., Segal, A., Vuik, C.: Modelling of multiphase flows with a Level-Set method. In: Feistauer, M., sí, V.D., Knobloch, P., Najzar, K. (eds.) Numerical Mathematics and Advanced Applications, pp. 698-707, ENUMATH 2003, Springer, Berlin (2004)
28. vander Pijl, S.P., Segal, A., Vuik, C., Wesseling, P.: A massconserving Level-Set method for modelling of multi-phase flows. Int. J. Numer. Methods Fluids 47, 339-361 (2005)

29. van Sint Annaland, M., Deen, N., Kuipers, J.: Numerical simulations of gas bubbles behaviour using a three-dimensional volume of fluid method. Chem. Eng. Sci. 60, 2999-3011 (2005)

30. van Kan, J.: A second-order accurate pressure correction method for viscous incompressible flow. SIAM J. Sci. Stat. Comp. 7, 870891 (1986)

31. Wesseling, P.: Principles of Computational Fluid Dynamics. Springer, New York (2001)

32. Yang, X., James, A., Lowengrub, J., Zheng, X., Cristini, V.: An addaptive coupled level-set/volume-of-fluid interface capturing method for unstructured triangular grids. J. Comput. Phys. 217, 364-394 (2006) 